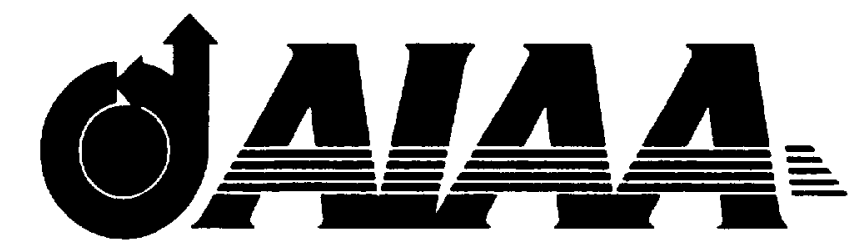

AIAA-2001-0907

Subsonic Reynolds Number Effects on a Diamond Wing Configuration (Invited)

J. M. Luckring

NASA Langley Research Center

Hampton, Virginia

T. A. Ghee

NAVAIR

Patuxent River, Maryland

39th AIAA Aerospace Sciences Meeting \& Exhibit 8-11 January 2001

Reno, Nevada 


\title{
Subsonic Reynolds Number Effects on a Diamond Wing Configuration
}

\author{
J. M. Luckring \\ Aerodynamics, Aerothermodynamics, and Acoustics Competency \\ NASA Langley Research Center \\ Hampton, Virginia \\ T. A. Ghee ${ }^{\dagger}$ \\ Advanced Aerodynamics Branch \\ NAVAIR \\ Patuxent River, Maryland
}

\begin{abstract}
An advanced diamond-wing configuration was tested at low speeds in the National Transonic Facility (NTF) in air at chord Reynolds numbers from 4.4 million (typical wind-tunnel conditions) to 24 million (nominal flight value). Extensive variations on high-lift rigging were explored as part of a broad multinational program. The analysis for this study is focused on the cruise and landing settings of the wing high-lift systems. Three flow domains were identified from the data and provide a context for the ensuing data analysis. Reynolds number effects were examined in incremental form based upon attached-flow theory. A similar approach showed very little effect of low-speed compressibility.
\end{abstract}

\begin{tabular}{ll} 
& \multicolumn{1}{c}{ NOMENCLATURE } \\
AR & wing aspect ratio, 2.2001 \\
b/2 & wing semispan, $2.6927 \mathrm{ft}$. \\
$C_{D}$ & drag coefficient \\
$C_{D .0}$ & zero-lift drag coefficient, planar wing \\
$C_{L}$ & lift coefficient \\
$C_{L .0}$ & lift coefficient at $\alpha=0^{\circ}$ \\
$C_{m}$ & pitching moment coefficient about \\
& $0.25 \mathrm{cbar}$
\end{tabular}

Senior Research Engineer, Subsonic Aerodynamics Branch, Associate Fellow, AlAA

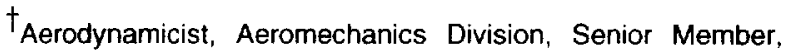
AIAA

Copyright (c) 2001 by the American Institute of Aeronautics and Astronautics, Inc. No copyright is asserted in the United States under Title 17, U. S. Code. The U. S. Government has a royalty-free license to exercise ali rights under the copyright claimed herein for Governmental Purposes. All other rights are reserved by the copyright owner. cbar wing mean aerodynamic chord, $3.1428 \mathrm{ft}$.

$c_{r} \quad$ wing root chord, $4.707 \mathrm{ft}$.

$C_{1} \quad$ wing tip chord, $0.1883 \mathrm{ft}$.

$\mathrm{K}_{\mathrm{p}} \quad$ attached-flow lift factor

$\mathrm{K}_{\mathrm{s}} \quad$ vortex-flow lift factor

$\mathrm{K}_{012}$ drag polar coefficients

$L_{\text {, }} \quad$ fuselage length, $7.7083 \mathrm{ft}$.

1 reference length

$M \quad$ Mach number

$P_{s} \quad$ percent suction

Rn Reynolds number ( $\mathrm{pul} / \mu$ )

$\mathrm{Rn}_{\mathrm{C}} \quad$ Reynolds number based on cbar

$\mathrm{S} / 2$ wing semi-area, $6.5908 \mathrm{ft}^{2}$

TNFA Typical Naval Fighter-Attack Vehicle

$\begin{array}{ll}\alpha & \text { angle of attack } \\ \Delta C_{i} & \text { increment in coefficient } C_{i}\left(C_{L}, C_{D}, \ldots\right) \\ \Lambda_{t} & \text { wing leading-edge sweep, 40 } \\ \lambda & \text { wing taper ratio, } C_{l} / C_{r}, 0.04\end{array}$

\section{INTRODUCTION}

High performance military aircraft represent a unique design challenge due to the inherently multi-mission nature of these vehicles (e.g., cruise vs. maneuver requirements, clean configuration vs. a variety of load-outs for external stores, etc.) The vehicle design must incorporate a compromise among many practical constraints and operational requirements. Recently, the requirement for low observability has added new constraints to vehicle shaping which results in a further compromise with aerodynamics and other disciplines for a successful aircraft design. 
Basic observability considerations can result in aggressive wing sweep angles in conjunction with reductions to wing twist and camber as well as leading-edge radius. These and other considerations can degrade wing aerodynamic performance itself, and lead to increased occurrences of flow separation. Reynolds number can be an important parameter to this issue. One particular operational consideration for these effects is the low-speed, high-lift conditions suitable to carrier approach.

Reynolds number, the ratio of inertial to viscous forces, remains the primary aerodynamic scaling parameter used to relate sub-scale wind tunnel models to full-scale airplanes in flight. Scaling technologies have been honed to a comparatively high degree of precision for the commercial transport community. Until recently, the accuracy requirements for Reynolds number scaling have not been as stringent for military fighter aircraft community, in part due to the inherent multimission nature of these vehicles. Even so, current budget constraints have increased the demand for first-flight certification of these vehicles against a variety of Key Performance Parameters (KPP). This can increase the accuracy requirements for a variety of factors that contribute to pre-flight performance prediction, one category of which is Reynolds number effects.

The extraction of Reynolds number effects is inherently problematic'. For example, there is always concern about the distinction between true and pseudo Reynolds number effects in a measurement process. With regard to the aerodynamics itself, care must be taken to identify the domains for various flow states (e.g., attached vs. separated) so as to distinguish changes in properties within a domain from changes of the domain boundary. An example of this type of analysis would be the various characteristics of aiffoil separation (see Polhamus ${ }^{2}$, for example). Finally, cost-benefit considerations must be weighed since the cost of high Reynolds number data exceeds that of conventional tunnel data. At the same time, the cost of a vehicle deficiency discovered in flight and resolved through flight test and fleet retrofit can certainly exceed the cost of ground-based testing capable of identifying such deficiencies.
The experimental program of the present study was a very extensive investigation of high-lift configuration variables, Reynolds number effects, and low-speed Mach number effects. The extent of data greatly exceeds that which can be analyzed within a given paper, and the focus for this paper is to begin an assessment of Reynolds number effects on a few of the configurations tested. Some initial analysis of the high-lift aerodynamics from this experiment has also been recently published. ${ }^{3}$

Emphasis here will be placed on the cruise wing aerodynamics to establish several flow domains developed by this wing and the associated Reynolds number effects. These results are then related to one high-lift configuration representative to carrier approach landing conditions. A brief review of the experimental process is presented first.

\section{EXPERIMENTAL PROCESS}

\section{Test Program and Objectives}

The test program was one part of a broader collaboration performed under the auspices of The Technical Cooperation Program (TTCP), a multinational research organization established to facilitate collaborative military research. Participants in this project were the Naval Air Systems Command (NAVAIR), NASA, and the Defense Evaluation and Research Agency (DERA) of the United Kingdom. The DERA contributions were sponsored by the British Ministry of Defense (MOD). This overarching collaboration involved design and fabrication of two wind tunnel models (the diamond wing of this report and a related "lambda" wing), wind tunnel testing in the DERA $5 \mathrm{~m}$ tunnel and the National Transonic Facility (NTF), and Computational Fluid Dynamics (CFD) assessments for these configurations.

The diamond wing test program was directed at assessment of an advanced slotted high lift system. The system was comprised of a full-span leading-edge flap, a slotted part-span trailing edge flap, and a flap preconditioner referred to as a shroud. High-lift rigging (gap and overlap) was optimized experimentally for a number of flap/shroud deflections, and the effects of Reynolds number and Mach number were assessed. 


\section{National Transonic Facility}

The NTF can be operated at Mach numbers ranging from 0.1 to 1.2 , at total pressures from 1.2 to 8.8 atmospheres, and at total temperatures from around $120^{\circ} \mathrm{F}$ down to minus $250^{\circ} \mathrm{F}$, the cryogenic temperatures being achieved through the evaporation of injected liquid nitrogen. The test section is 8.2 feet square and approximately 25 feet long. The basic facility circuit is shown in Figure 1.

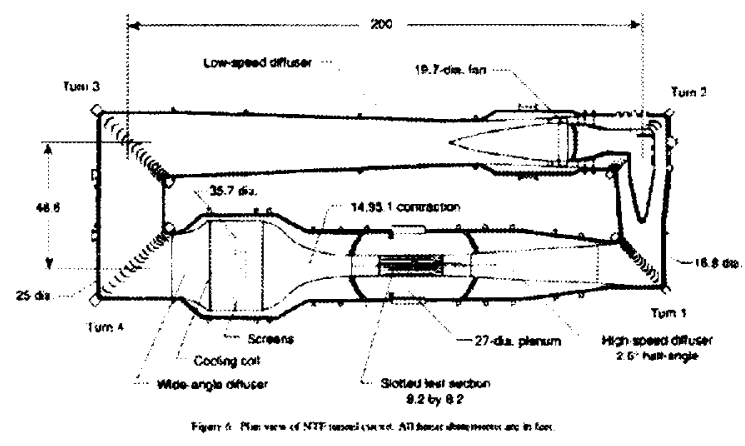

Fig. 1- NTF Circuit

An overall Mach-Reynolds number facility envelope is presented in Figure 2 for NTF (both air and cryogenic modes) and compared with several other facilities. The reference length for the facility envelopes is one-tenth of the square root of the test-section area. Also shown for reference are some operating conditions for a variety of slender vehicles as well as a typical High-Wing Military Transport (HWMT).

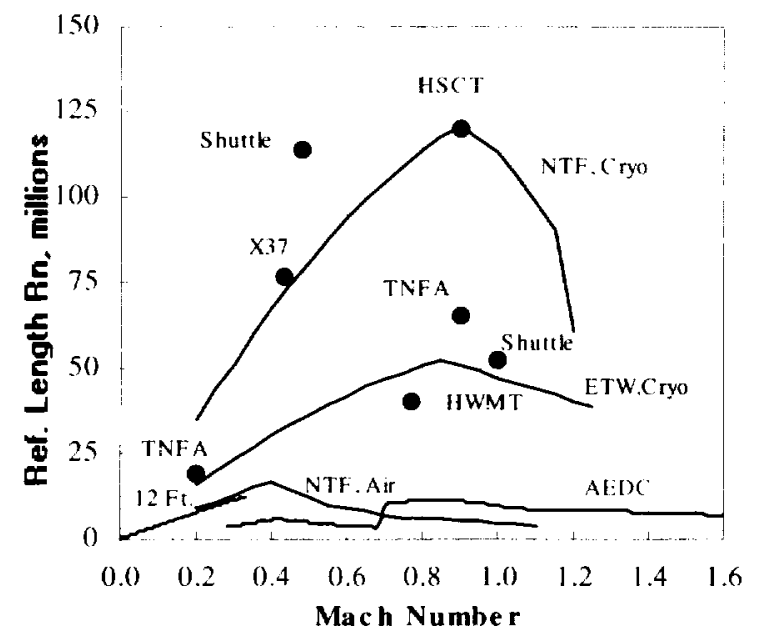

Fig. 2 - Facility Envelopes
Beginning at two-tenths Mach number for air the NTF and the 12-Ft tunnel essentially overlap. Additional details of the facility can be found in references 4 and 5 . Several recent reviews ${ }^{67}$ of NTF research activity have been published.

For this particular test program, the facility was used as a conventional pressure tunnel. The model had been designed for conventional pressure tunnel testing, and a loads assessment showed that it could not be tested under cryogenic conditions due to wing spar limitations. The facility supports both full-span and semispan testing, and this test used the semispan test capability.

Conventional operations for the facility include testing in a slotted test section. For this particular program the customers were interested in testing in a solid-wall test section. Although the facility has had slot covers for this purpose, this was the first such test, and a separate facility calibration was completed for the solid-wall test section at the test conditions of interest to the diamond wing program.

\section{Wind Tunnel Model}

The model was a semispan configuration mounted on the side wall of the test section. It was comprised of a stand-off, fuselage, wing, and highlift components. A photograph of the model with the cruise wing is shown in Figure 3, and some overall configuration dimensions are included in the nomenclature.

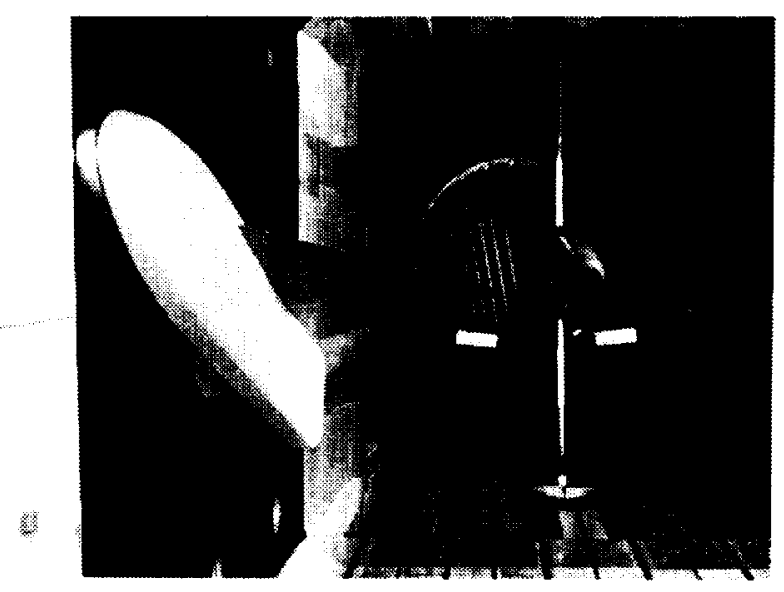

Fig. 3 - Diamond wing model in NTF. 
The combined effects of semispan testing and low aspect ratio resulted in a relatively large reference chord. As a consequence, data representative of full-scale flight conditions could be obtained with the facility operating around 6 atmospheres. The NTF-air envelope scaled for the diamond wing model is shown in Figure 4 and is compared to nominal carrier approach conditions for a Typical Naval Fighter-Attack vehicle.

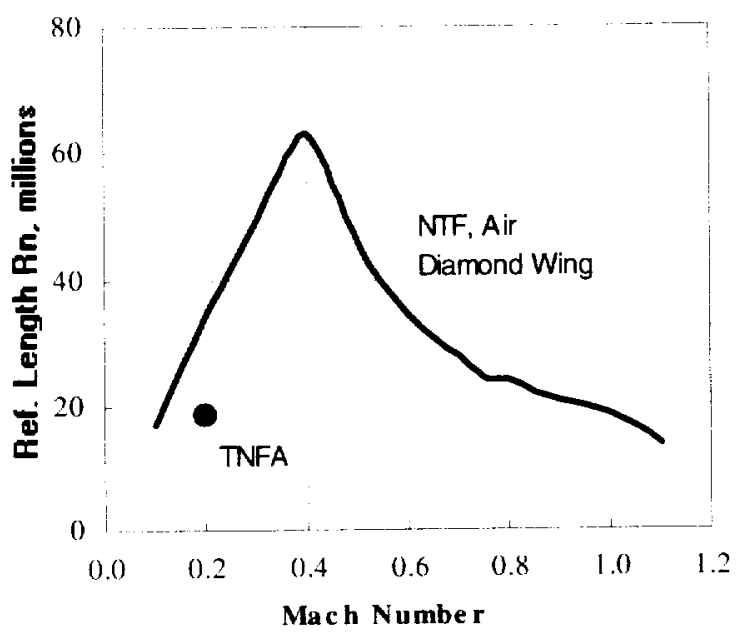

Fig. 4- Facility envelope for diamond wing in NTF. Air mode.

The cruise wing had $40^{\circ}$ swept leading and trailing edges, was thin, and incorporated a number of practical design considerations for military operations. At the leading edge the wing had a full-span constant-chord hinged flap with the hingeline also swept at $40^{\circ}$. At the trailing edge the wing had a part-span constant-chord slotted flap and a flap shroud to precondition the flow approaching the trailing edge flap. The leading-edge flap could be deflected at $0^{\circ}$ or $22^{\circ}$, the shroud at $0^{\circ}, 17^{\circ}$ or $23^{\circ}$, and the trailing-edge flap at $0^{\circ}, 20^{\circ}$ or $35^{\circ}$. Figure 5 shows the high-lift system.

The model provided for systematic variation of the trailing-edge flap gap and overlap. The notional gap settings were $0.5,1.5$, and 2.5 percent chord, and the notional overlap settings were $-1.0,0.5$, $1.5,2.0$, and 3.0 percent chord. Positive overlap was defined for the leading edge of the flap being upstream of the trailing edge of the shroud. The model was designed to provide for intermediate settings of these parameters.
The fuselage was a generic design with a Dshaped cross section and a flat side to facilitate sealing the fuselage/high-lift juncture. A transition strip was affixed to the fuselage nose, and the fuselage base was flat to fix separation for all Reynolds numbers tested. The model was mounted on a non-metric side-wall stand-off, or plinth, to manage wind-tunnel side-wall boundary layer interactions. The stand-off was 1.5 inches wide, and the interface between these

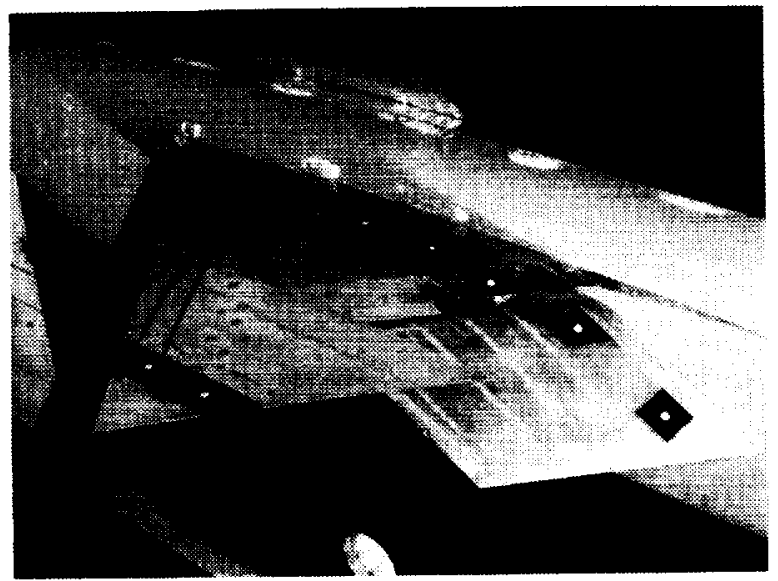

Fig. 5- High lift system.

components was a labyrinth seal to mitigate flow leakage into the fuselage. The wing/fuselage geometry was mounted on the NTF $114 \mathrm{~S}$ semispan balance for force and moment measurements. The model was also instrumented with about 450 static surface pressure orifices. All aspects of this semispan installation were within the guidelines of a semispan test technology activity ${ }^{8-10}$ underway at LaRC.

The particular setting for the configurations of this study are summarized in the table below

\begin{tabular}{|l|l|l|l|l|l|}
\hline Name & Lef & Shrd & Tef & Gap & Ovlp \\
\hline Cruise & 0 & 0 & 0 & 0 & 0 \\
\hline Landing & $22^{\circ}$ & $23^{\circ}$ & $35^{\circ}$ & $1 \%$ & $0.5 \%$ \\
\hline Take off & $22^{\circ}$ & $0^{\circ}$ & $20^{\circ}$ & 0 & 0 \\
\hline
\end{tabular}

Test Conditions, Procedures and Data Reduction

Test Conditions - Data were obtained at a fixed total temperature of $95^{\circ} \mathrm{F}$ and at total pressures ranging from 1.1 to 6 atmospheres. Tests were conducted for Mach numbers ranging from 0.1 to 0.35 . This resulted in Reynolds numbers that 
varied from 4.4 to 24 million based on the cruise wing mean aerodynamic chord. Angle of attack varied from -5 to 24 degrees. The test took place from July to October of 1999.

Additional gap and overlap settings were tested to help identify the optimum high-lift rigging. Nominal repeat runs were also included to address data quality and repeatability. ${ }^{11} 12$

Aeroelastic Deformation - Static aeroelastic deformation can always be a consideration in wind tunnel data. Although this is usually not as great a concern for low aspect-ratio configurations, it could still be a factor in high-lift testing. In particular, the relative motion of the flap with respect to the wing could have effects on the gap flow of the high-lift system.

To quantify these effects, an optical aeroelastic measurement system was used to determine both absolute deflections and the relative deformation of the high-lift system with respect to the wing. .The method used single-camera videogrammetry and was the first such application to a semispan model in NTF. ${ }^{13}$ Several of the surface targets for these measurements can be seen in Figure 5 . Measurements were performed for a selected subset of the entire test matrix, and the measured deformations and effects on the high-lift gap were found to be small. Thus, it is inferred that model static aeroelastic effects in the data set are small.

Solid-wall Interference - The test was conducted with solid wall test section. Although NTF testing typically is performed with slotted test section, there was concern for this test that the highly deflected wing wake could interact with the floor slot flow. Such an interaction would be difficult to correct for, and would also be a potential source for pseudo Reynolds number effects. Therefore, the test was conducted with the test section configured to solid walls.

The data have been assessed for solid-wall interference effects with classical interference techniques $^{14.15}$ (both with and without a Maskell separated wake correction) and also with a Wall Interference Correction System (WICS) that utilizes both model loads and wall pressures. ${ }^{16.17}$ The WICS methodology as implemented in NTF is considered to be the baseline representation for wall-induced effects, and was used for the final data reduction process. The other techniques, such as full Maskell, are also available for data reduction.

\section{RESULTS}

Analysis is primarily presented for the cruise wing configuration. Here the various domains of attached and separated flow are identified, and then Reynolds number and compressibility effects are discussed. Similar results were found for both of the take-off and landing configurations analyzed but with the attached-flow regime offset in angle of attack due to the various device settings. Some representative results for the landing configuration are also included.

\section{Cruise Configuration}

Basic Aerodynamic Properties- This analysis will be focused on the high Reynolds number data, $\mathrm{Rn}_{\mathrm{c}}$ $=24.2 \times 10^{6}$ at a reference carrier approach Mach number of 0.2. Guidance to discern flow states can be obtained by first examining drag as a function of lift squared, Figure 6 . At the lowest loading conditions (Figure $6 \mathrm{a}, 0<\mathrm{C}_{\mathrm{L}}^{2}<0.05$ ), the data show a very linear trend, implying the flow to be attached for this range. This is followed by an abrupt break in the curve (filled symbol) to a second linear trend over moderate loading conditions. The abruptness of this break would imply an abrupt flow separation, the details of which will be discussed subsequently in the context of overall forces and moments. Theoretical estimates for the data are also shown based upon linear least-squares fits within the respective low- $\alpha$ and mid- $\alpha$ ranges.

In Figure $6 \mathrm{~b}$, the full data range is shown along with the low- $\alpha$ and mid- $\alpha$ theories. Here a second but softer break from the mid- $\alpha$ theory is seen, around $\mathrm{C}_{\mathrm{L}}^{2}=0.5$, and is also tagged by a filled symbol. This drag rise increment for $C_{L}^{2}>0.5$ is more quadratic in nature and implies a change in the character of the separated flow.

Overall force and moment coefficients are shown in Figure 7 along with some theoretical estimates and the two break point conditions discussed above. The theoretical estimates model the attached-flow properties for this configuration. The attached-flow lift can be theoretically approximated by

$$
C_{L}=C_{L, O}+K_{p} \cos ^{2} \alpha \sin \alpha
$$


The coefficients in the equation were obtained through a linear least squares fit of the data for the functionality of the above equation. The

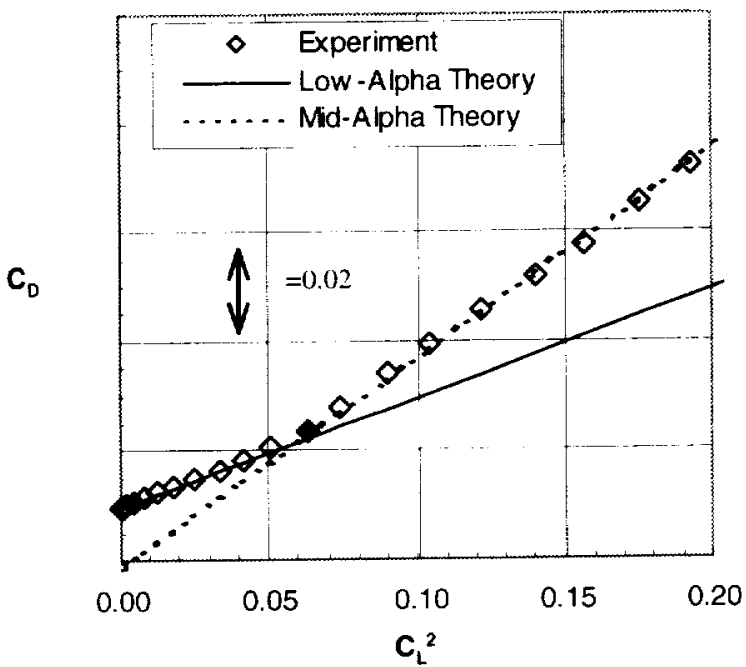

a) Low- $\alpha$ range

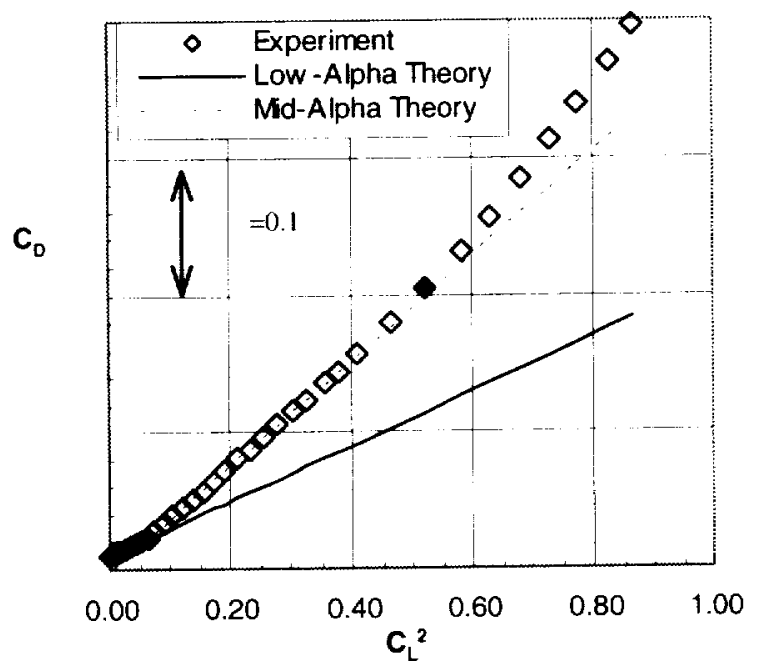

b) Full- $\alpha$ range

Fig. 6- Drag analysis. $M=0.2, R n_{c}=24.2 \times 10^{6}$, Cruise Config.

results of this fitting process were relatively insensitive to the range of data included in the analysis as long as the range did not exceed that identified as exhibiting attached-flow properties (i.e., $C_{L}^{2}<0.05$ or $C_{L}<0.22$ ). This particular equation neglects the lift contribution from the

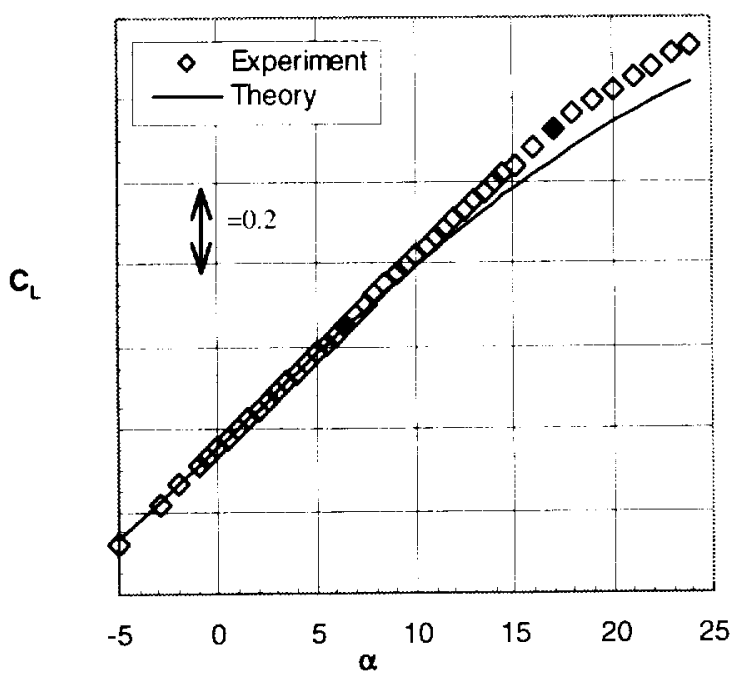

a) Lift

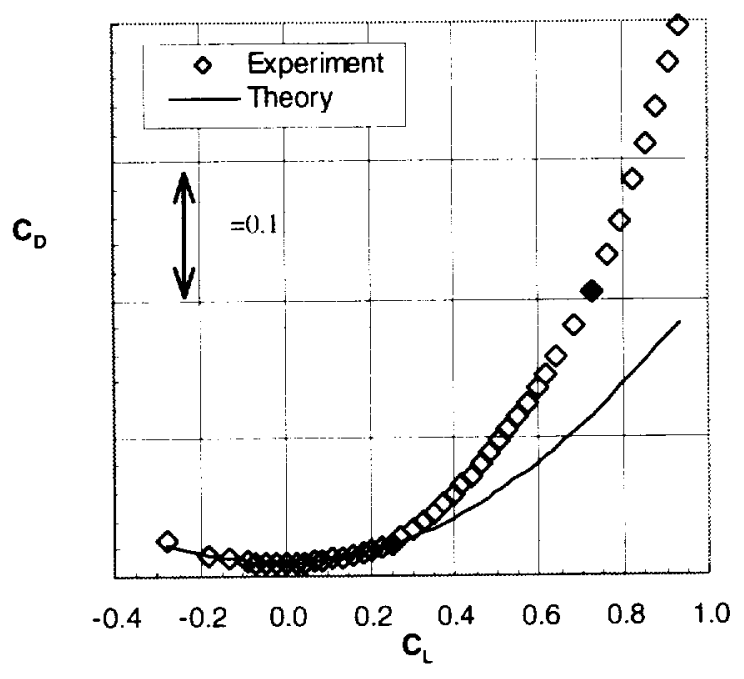

b) Drag

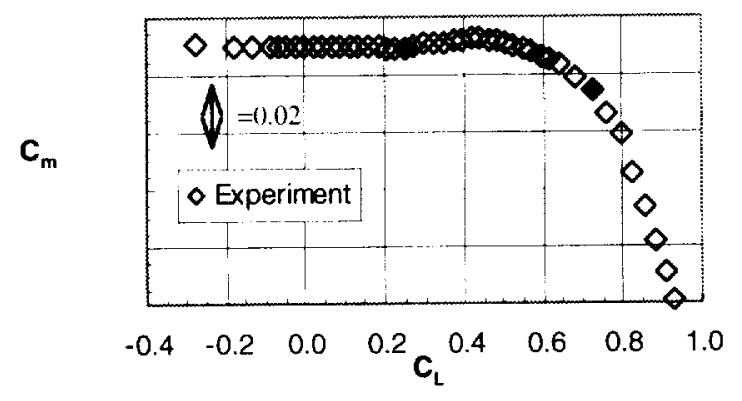

c) Pitching moment

Fig. 7- Basic forces and moments. $M=0.2$, $\mathrm{Rn}_{\mathrm{c}}=24.2 \times 10^{6}$, Cruise Config.

6

American Institute of Aeronautics and Astronautics 
leading edge suction since it is proportional to $\sin ^{3} \alpha$ and hence extremely small at the conditions of the analysis. However, the proper high- $\alpha$ formulation is included to allow for the assessment of incremental effects between the data and the attached-flow theory at these conditions.

In a similar vein, attached-flow estimates for the drag polar are modeled theoretically with

$$
\mathrm{C}_{\mathrm{D}}=\mathrm{K}_{0}+\mathrm{K}_{1} \mathrm{C}_{\mathrm{L}}+\mathrm{K}_{2} \mathrm{C}_{\mathrm{L}}^{2}
$$

and the coefficients are again achieved from a linear least squares fit for the attached-flow domain of the data. The linear term is included since the wing has twist and camber; for the same reason the term $\mathrm{K}_{0}$ should not be confused with $\mathrm{C}_{0.0} . \mathrm{K}_{2}$ is the coefficient of induced drag and will be used in later analysis.

Examination of the data of Figure 7 in the context of the identified break points and attached-flow theoretical estimates is quite revealing. Looking at the lift first, it is seen that at the first break point, around $\alpha=6^{\circ}$, the lift actually increases and continues to exceed the attached-flow estimate for the full angle of attack range. This would imply a leading-edge vortex type of flow separation. The drag polar also shows the abrupt break from the attached-flow estimate, which is consistent to the results of Figure 6.

There is also an abrupt change in the pitching moment trends at the first break point. Up to the break point, the pitching moment is linear with lift and has a very slightly stable slope. At the break point, the pitching moment abruptly shows a positive, or nose-up increment. This indicates that the additional lift associated with the flow separation is being realized ahead of the moment reference point and is consistent with the onset of a leading-edge vortex.

As angle of attack increases beyond the first break point the vortex will gain strength and also sweep inboard over the wing. This can result in the inverted parabolic shape of the pitching moment curve between the two break points.

The sweep of this wing is relatively low with respect to a leading-edge vortex flow, and there are several consequences. ${ }^{18}$ The vortex will be relatively strong and could exhibit vortex breakdown characteristics with only moderate increases in angle of attack beyond that of incipient separation. Compared to the attachedflow lift, the vortex lift increment will be smaller than the increment for slender delta wings.

Analysis is further aided by examining the data in incremental form. A lift increment, $\Delta C_{L}$, is formed by subtracting the attached-flow theoretical estimate from the experimentally measured result. The results are presented in Figure 8.

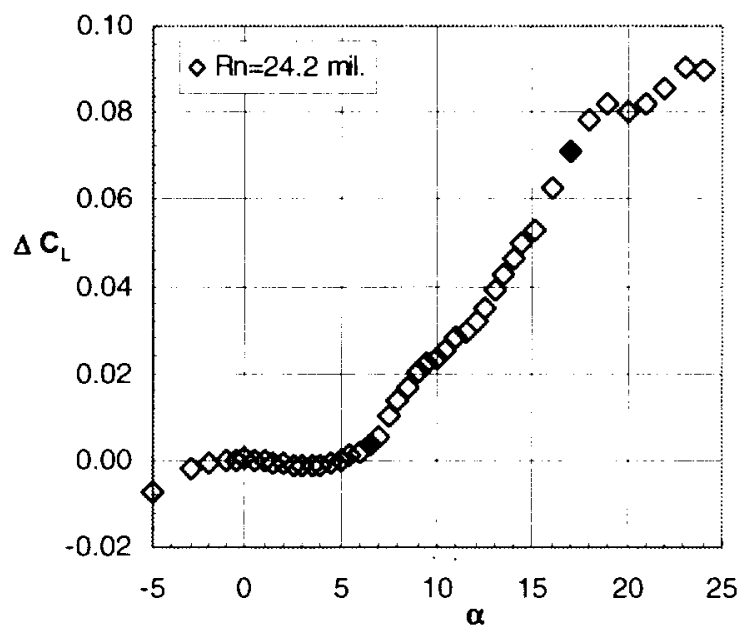

Fig. 8 - Lift increment, experiment - theory. $M=0.2, R n_{c}=24.2 \times 10^{6}$, Cruise Config.

Significantly more detail in the lift characteristics can be seen in this figure. It appears that the onset of the vortex lift is evidenced around $\alpha=4^{\circ}$, slightly before the first break point from the drag analysis. The initial build up of the lift increment is roughly quadratic with the angle of attack increment beyond insipient separation as would expected from vortex lift theory ${ }^{19}$

$$
C_{L}=C_{L, O}+K_{p} \cos ^{2} \alpha \sin \alpha+K_{v} \sin ^{2}\left(\alpha-\alpha_{S}\right) \cos \alpha
$$

$K_{v}$ is the usual vortex-lift coefficient and the equation is expressed to approximate leadingedge separation at $\alpha=\alpha_{s}$. The quadratic lift build up is only sustained up to $9^{\circ}$ or so where the character of the lift increment changes, probably in association with some sort of disorganization of the leading edge vortex. 
The second break point (around $\alpha=17^{\circ}$ ) occurs close to another change in the lift increment. The wing seems to be approaching more of an overall stall condition. Returning to Figure 7 it is seen that the second break point coincides with a departure from linear lift growth and the continued build up of negative pitching moments. This would not be inconsistent with the onset and progression of vortex-induced lift losses.

The basic flow structure inferred for each domain is substantiated by experimental pressure distributions

Reynolds Number Effects - The foregoing analysis is carried over to assess Reynolds number effects for the cruise wing. For the most part, data will be presented for the low, moderate, and high Reynolds numbers tested. This spans conventional-tunnel to representative-flight conditions. Intermediate Reynolds number data will be used for trend analysis

Lift data are presented in Figure 9 for three of the six Reynolds numbers tested. These data span the full angle of attack and Reynolds numbers range tested, and as such Reynolds number effects are difficult to discern. Also shown is the high Reynolds number attached-flow theory from the previous figures.

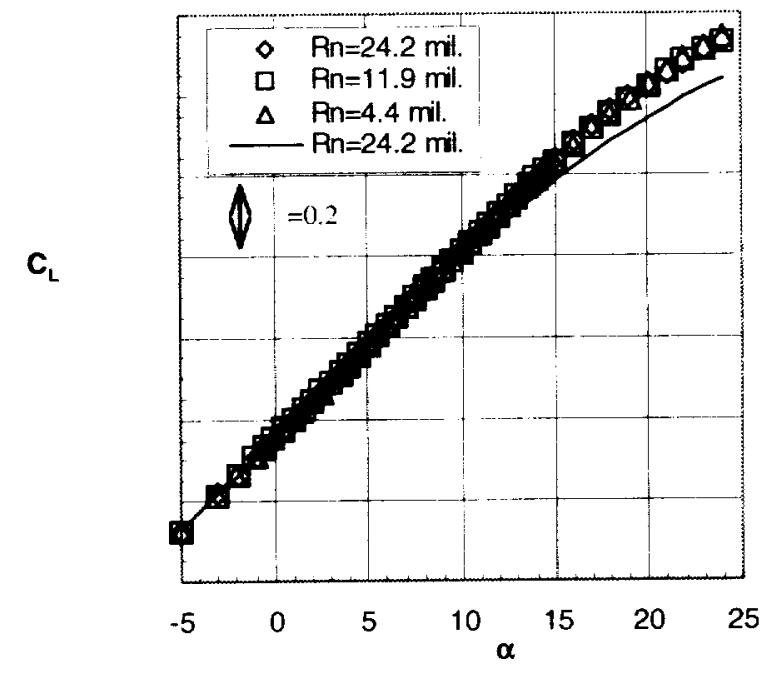

Fig. 9- Lift data for several Reynolds numbers. $M=0.2$, Cruise Config.
The same analysis approach of Figures 7 and 8 was adopted for the data of Figure 9. Each Reynolds number condition was individually fit to establish its attached flow characteristics. The resulting lift increments between the experiment and the attached-flow theory are shown in Figure 10. Looking first at the low- $\alpha$ range, it is seen that onset of separation is delayed with increasing Reynolds number, from roughly $4^{\circ}$ to $6^{\circ}$. A similar trend is seen for the negative angles of attack, with the lowest Reynolds number data separating first.

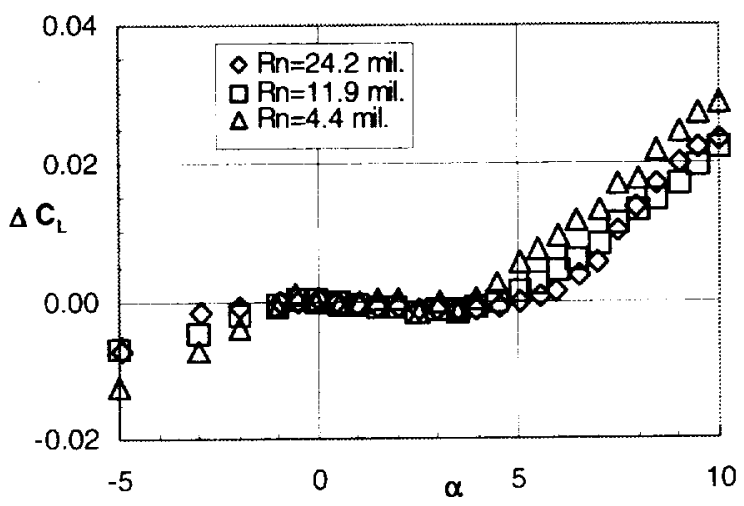

a) Incremental effects - low $\alpha$

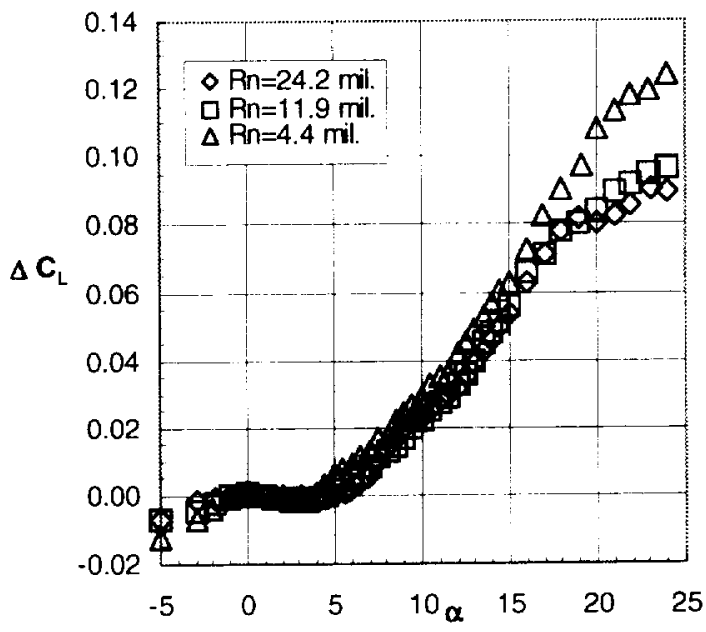

b) Incremental effects - full range

Fig. 10- Reynolds number effect on lift increment. $M=0.2$, Cruise Config. 
Looking at the full range of data, it is seen that the lowest Reynolds number data do not evidence the lift loss break in $\Delta \mathrm{C}_{\mathrm{L}}$ that the other Reynolds number data do around $18^{\circ}$ angle of attack. The reason for this is unclear. However, this means that the low Reynolds number data are not conservative with regard to lift, that is, at flight values of Reynolds number the high angle of attack $C_{L}$ is about 0.03 less than the value measured at conventional wind tunnel conditions. Additional data analysis has indicated the lowest Reynolds number data to be different in many respects from the data obtained at $R n_{c}=12 \times 10^{6}$ or beyond.

As one example, Reynolds number effects on the pitching moment characteristics are shown in Figure 11. The low Reynolds number data show an unstable pitch trend for $0<C_{L}<0.4$. The moderate and high Reynolds number data show rather similar trends with neutral to slightly stable pitching moments up to a $C_{i}$ of about 0.25 where the flow separates.

Drag polars for the range of Reynolds numbers tested are presented in Figure 12. The attachedflow fit to each data set is also included, and the results are shown only up to moderate lift levels, $C_{L}$ $<0.4$.

Before analyzing the attached-flow portion of these polars, the separated-flow drag increments are presented in Figure 13. As with the lift, these increments are taken between the measured result and the attached-flow theory for each Reynolds number. For the range of Reynolds numbers tested the separation tends to occur at $C_{L}=0.25$. Drag increments due to flow separation are more severe at the high Reynolds numbers. Data from the intermediate Reynolds numbers tested are consistent with these trends.

Returning to the attached-flow portion of the polar, it is seen in Figure 12 that the theoretical fit models the data very well in this domain. There are two Reynolds number effects to be discussed with these data. The first is a reduction in minimum drag with increasing Reynolds number, an overall trend to be expected. The second is a decrease in drag due to lift with increasing Reynolds number, a perhaps less anticipated result.
These attached-flow trends are quantified by analyzing the parameters of the attached-flow theoretical fit to the data as a function of Reynolds number. Here $C_{D}$, will be used instead of $K_{0}$. The values for $\mathrm{C}_{\mathrm{D} .0}, \mathrm{~K}_{1}$, and $\mathrm{K}_{2}$ are presented as a function of Reynolds number in Figure 14.

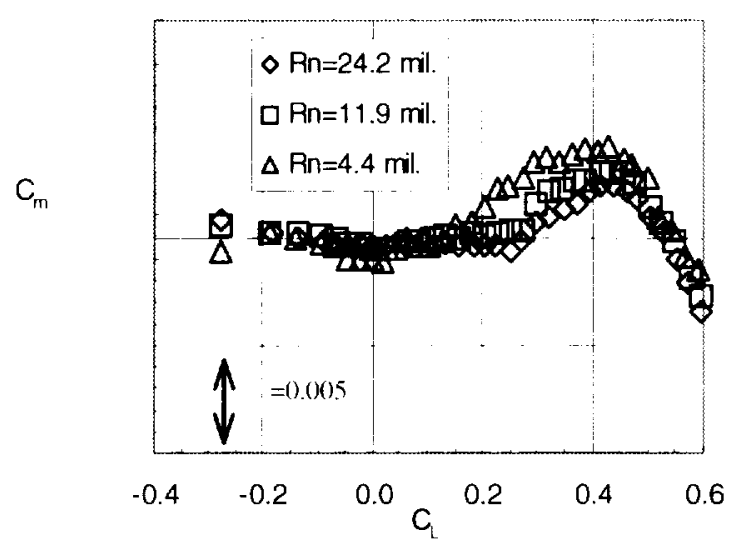

Fig. 11- Pitching moment. $M=0.2$, Cruise Config.

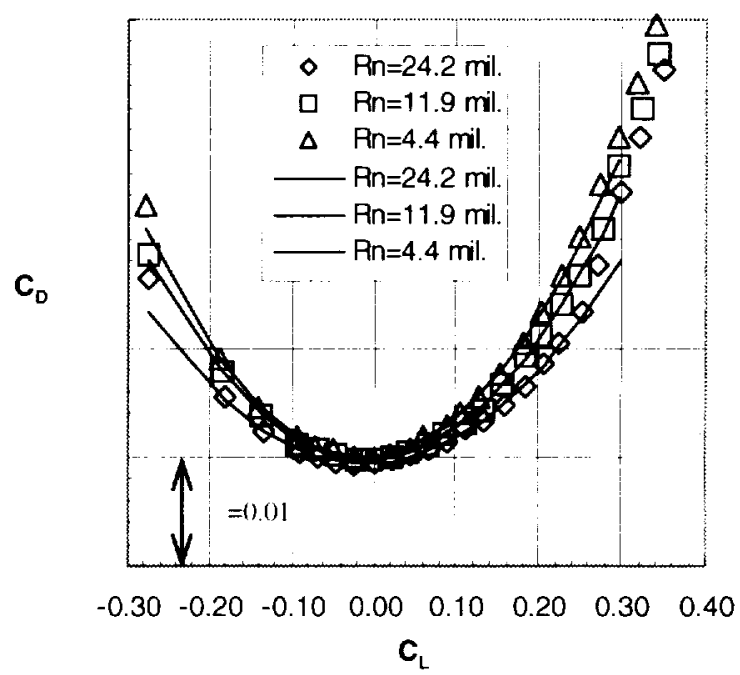

Fig. 12- Drag polars. $M=0.2$, Cruise Config.

The reduction in $\mathrm{C}_{0}$ follows a power-law trend with Reynolds number from the moderate to high values tested. The drop is much less than would be expected for $a-1 / 7$ power law relationship. 


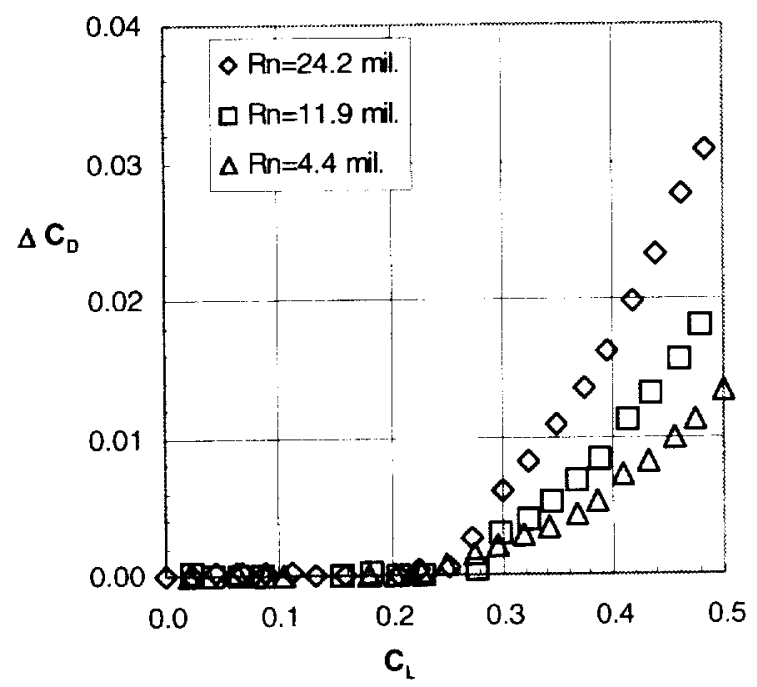

\section{a) Separation onset}

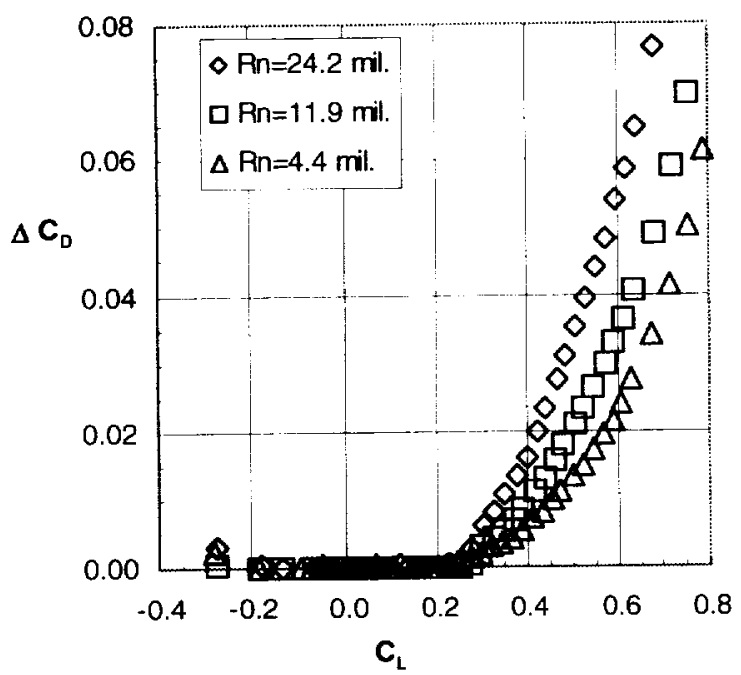

\section{b) Separation progression.}

Fig. 13- Drag increment. $M=0.2$, Cruise Config.

However, current estimates for the data uncertainty of $C_{D, o}$ is approximately a couple of counts $^{20}$ and this uncertainty band would include power law relationships closer to that expected for classical skin-friction variation with Reynolds number.

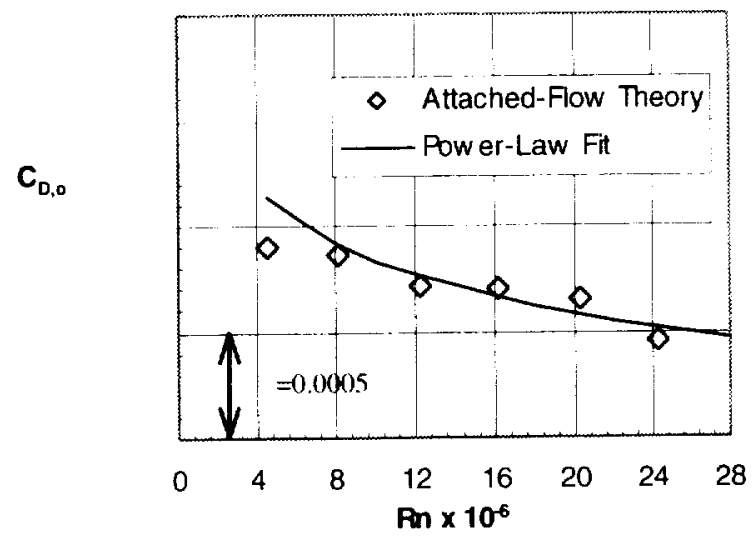

a) Skin friction

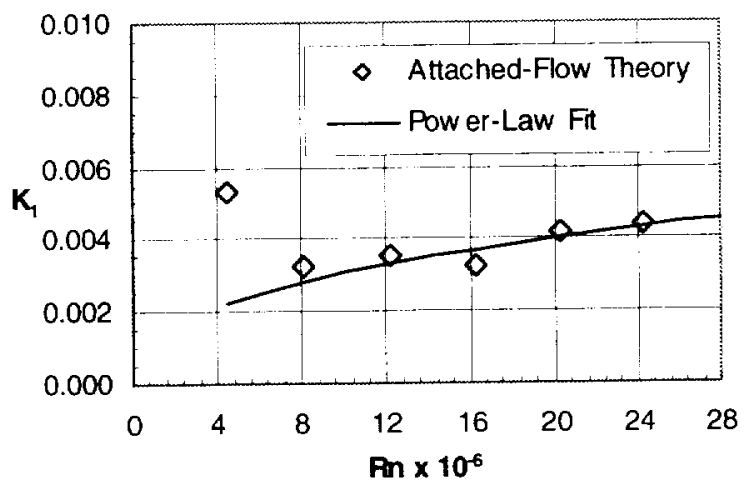

b) Skewness

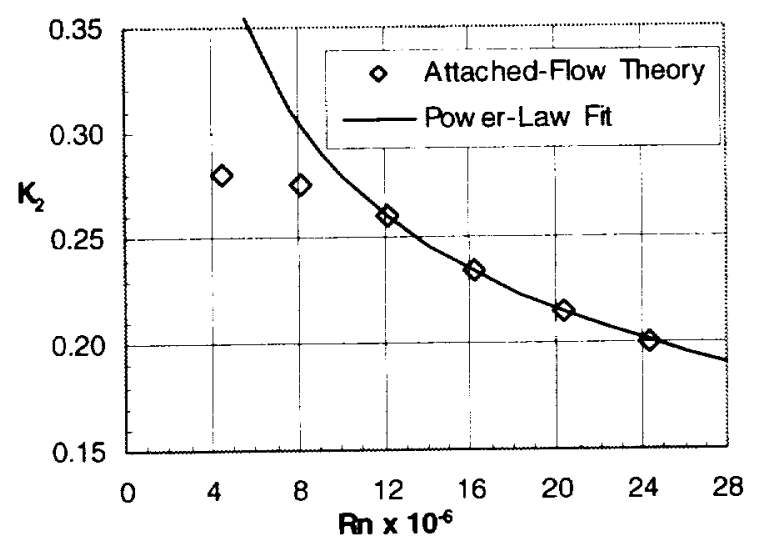

c) Drag due to lift

Fig. 14- Reynolds number effects on attachedflow drag polar parameters. $M=0.2$, Cruise Config. 
$\mathrm{K}_{1}$ is a measure of the skewness of the drag polar. The magnitude of this parameter for the cruise diamond wing is small and the variation with Reynolds number is also small. The low magnitude of $\mathrm{K}$, reflects the fact that the cruise wing geometry has a relatively small amount twist and camber.

The variation of $\mathrm{K}_{2}$ with Reynolds number is noteworthy. $K_{2}$ is the coefficient of induced drag and this drops from a value of 0.28 to 0.20 over the Reynolds number range tested. Based on the low Reynolds number result, this is a reduction of approximately $29 \%$, and here again the values of $\mathrm{K}_{2}$ at higher Reynolds numbers follow a power law relationship. For reference, this diamond wing has $1 / \pi \mathrm{AR}=0.145$.

Very similar trends were reported by Henderson ${ }^{21}$ in 1966 and presented in terms of percent suction. The incremental drag polars, with the skin friction drag removed, are shown in Figure 15a along with both the zero and one-hundred percent suction estimates. The data are seen to be well bounded by the two theoretical limits. The resultant estimates for percent suction are shown in Figure 15b. These results show that, at low lift levels, the wing developed roughly $50 \%, 60 \%$, and $80 \%$ suction for attached flow at the three Reynolds numbers analyzed. This is followed by conventional trends as flow separation effects were manifested. The results of the present study appear to be consistent with those reported by Henderson. ${ }^{21}$

The Reynolds number trends of Figure 14 further demonstrate that the lowest Reynolds number data develop flow characteristics that are atypical of the high Reynolds number data. For this wing, it appears that a Reynolds number of about 12 million is sufficient for scaleable results based upon power-law formulations. This is about half of the representative full-scale Reynolds number.

Mach Number Effects - As a secondary objective, the effects of compressibility on the low-speed aerodynamics was examined for a reduced portion of the test program. Data were obtained at Mach numbers ranging from 0.10 to 0.35 and, for the most part, these effects were determined to be small or predictable. In the interest of space, the analysis of these effects will not be presented.

\section{Landing Configuration}

A similar analysis for the high-lift landing configuration was performed. The principle effect of the deployed high-lift system was to shift the angle-of-attack and lift range over which the configuration exhibited attached flow. Selected Reynolds number effects will be presented to highlight this feature.

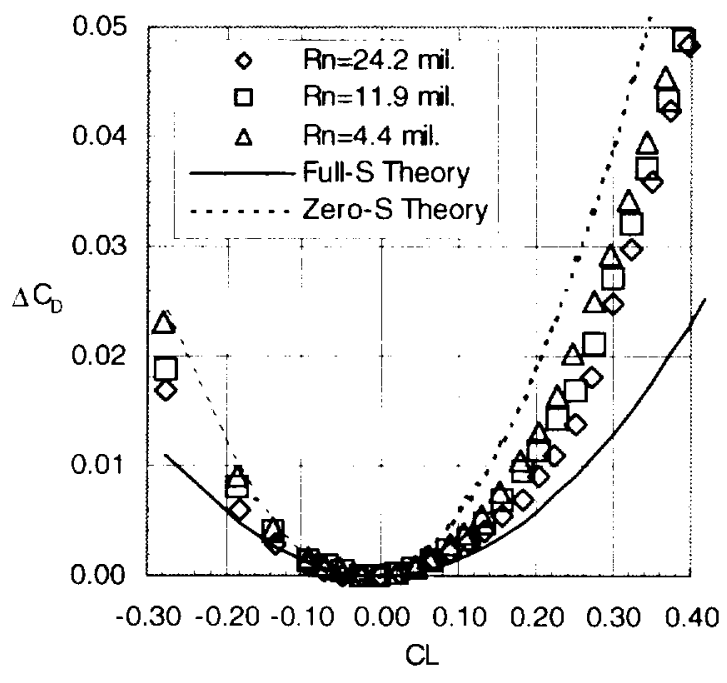

a) Drag bounds

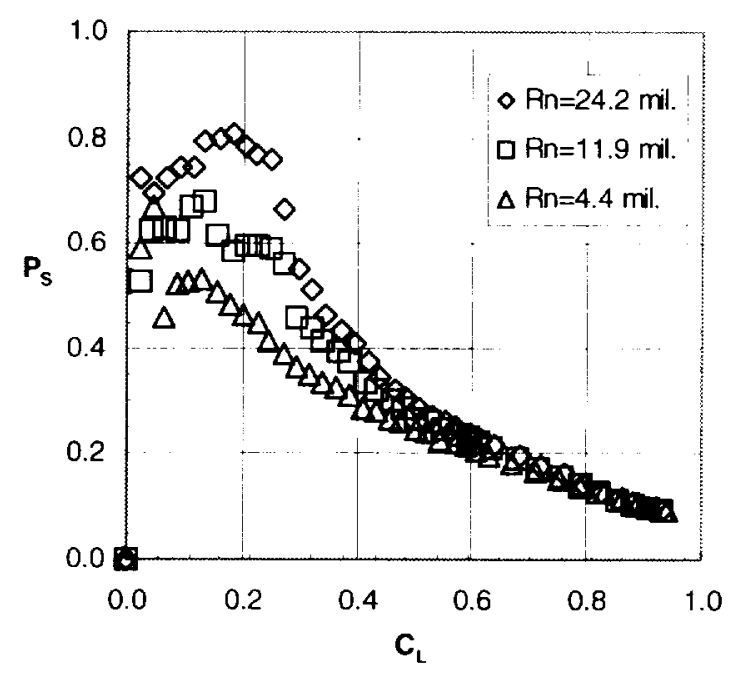

b) Percent Suction

Fig. 15- Percent Suction. $M=0.2$, Cruise Config. 
Reynolds Number Effects - The basic lift curve for the same three Reynolds numbers of the cruisewing analysis presented in Figure 16 along with

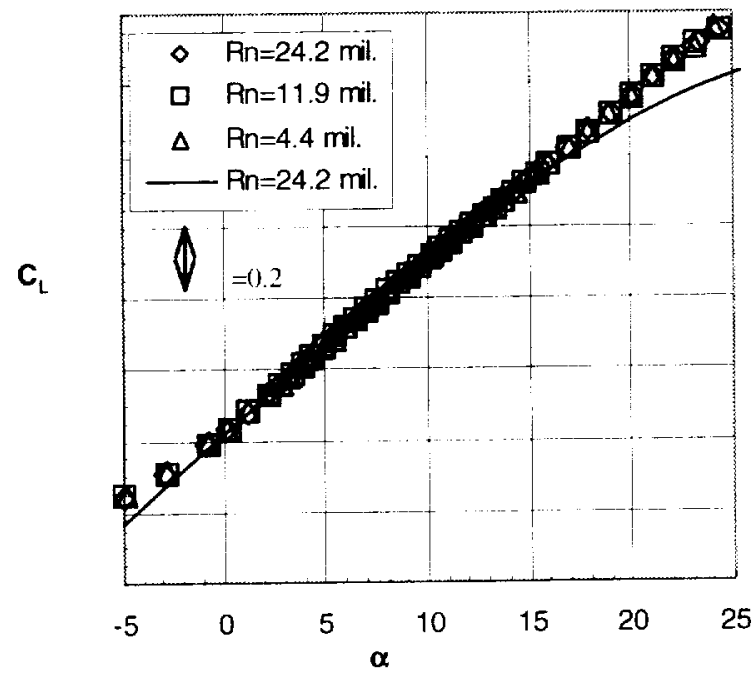

Fig. 16- Reynolds number data. $M=0.2$, Landing Config.

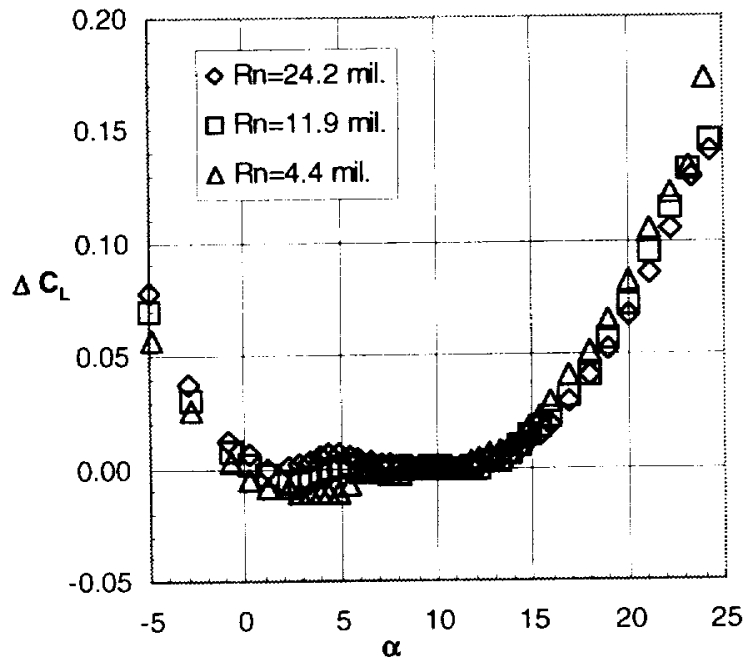

Fig. 17- Lift Increment. $M=0.2$, Landing Config.

the reference attached-flow theory for the high Reynolds number case. The data show an attached-flow region centered roughly around $\alpha=7^{\circ}$. This can be seen in Figure 17 where the lift increment is presented. Again, the data for each Reynolds number was individually fit to establish the theoretical attached-flow trends.
For this configuration the flow appears to stay attached for roughly $5^{\circ}<\alpha<10^{\circ}$. Separated flow effects are seen both below and above this range. For comparison, the Cruise Configuration exhibited separation around $\alpha=5^{\circ}$.

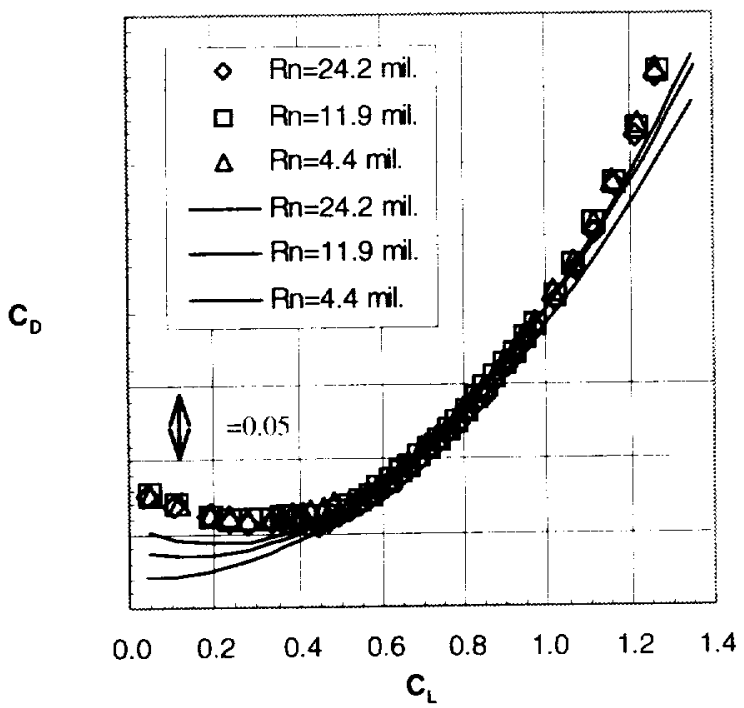

Fig. 18- Drag data. $M=0.2$, Landing Config.

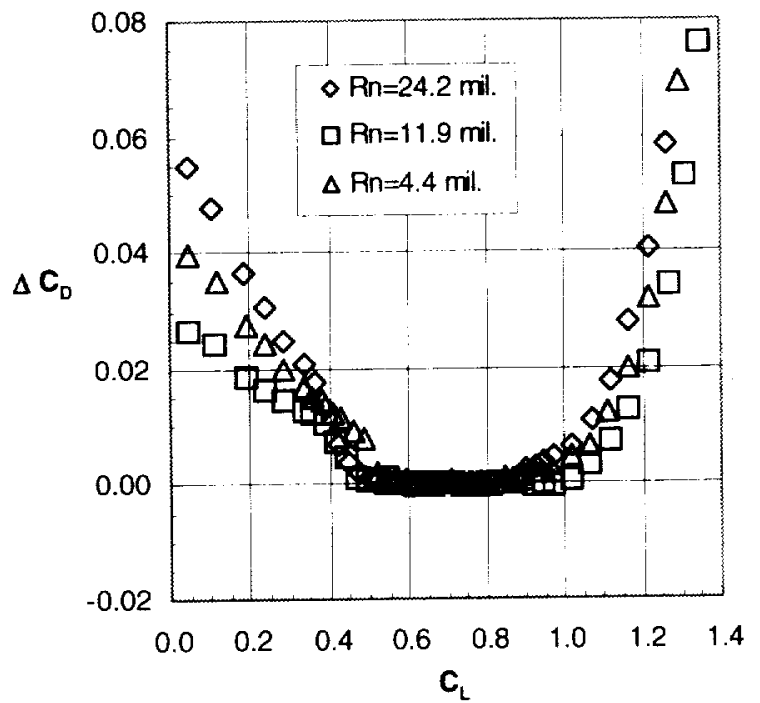

Fig. 19- Drag increments. $M=0.2$, Landing Config. 
Drag polars are presented in Figure 18. The attached-flow drag polar theory is presented for all three Reynolds numbers shown. Again, the attached-flow region is evident. The drag increments between experiment and theory further demonstrate this aspect, Figure 19. Here the attached-flow region is seen to extend roughly for $0.5<\mathrm{C}_{\mathrm{L}}<0.9$.

\section{CONCLUSIONS}

An assessment of the aerodynamic properties and Reynolds number effects has been presented for an advanced diamond-wing configuration. The model was tested in the NTF using total pressure to change Reynolds number. The focus for this study was the cruise wing geometry with limited discussion of a high-lift landing configuration.

Basic analysis showed that the cruise wing exhibited three flow domains. The flow remained attached up to approximately $5^{\circ}$ angle of attack after which a leading-edge vortex flow was manifested. Above $17^{\circ}$, the wing began to exhibit approach to stall and maximum lift. The data clearly showed an increase in Reynolds number delayed wing separation by two or three degrees.

Data were obtained at Reynolds numbers ranging from nominal atmospheric conditions (4.4 million) to representative flight values (24.2 million). The lowest Reynolds number data exhibited a number of differences from the moderate to high Reynolds number results. From this analysis, it seemed as a Reynolds number of 12 million was sufficient for the data to follow simple power-law scaling relationships.

The largest Reynolds number effect reported was on attached-flow drag due to lift. Wing efficiency went from roughly 50 percent suction to 80 percent suction with Reynolds number increase. This trend is consistent with prior experimental findings.

\section{REFERENCES}

'Elsenaar, A., Binion, T. W. Jr., and Stanewsky, E., "Reynolds Number Effects in Transonic Flow," AGARDograph AG-303. December 1988.

${ }^{2}$ Polhamus, E. C., "A Survey of Reynolds Number and Wing Geometry Effects on Lift Characteristics in the Low Speed Stall Region," NASA CR-4775, June 1996.

${ }^{3}$ Ghee, T. A., and Taylor, N. J., "Low-Speed Wind Tunnel Tests on a Diamond Wing High-Lift Configuration," AIAA Paper 004507, August 2000
${ }^{4}$ Fuller, D. E., "Guide for Users of the National Transonic Facility," NASA TM-83124, 1981.

${ }^{5}$ Foster, J. M., and Adcock, J. B., "Users Guide for the National Transonic Facility Research Data System," NASA TM-110242, April 1996.

${ }^{6}$ Wahls, R. A., "The National Transonic Facility - A Research Retrospective," AIAA Paper 01-0754, January 2001.

ZLuckring, J. M., “An Overview of National Transonic Facility Investigations for High Performance Military Aerodynamics (Invited)" AIAA Paper 01-0906, January 2001.

${ }^{8}$ Gatlin, G, Parker, P., and Owens, L. R., Jr., "Status of the Development of a Semi-span Test Capability at the National Transonic Facility," AIAA Paper 01-0759, January, 2001.

${ }^{9}$ Gatlin, G. M., and McGhee, R. J,: Experimental Investigation of Semispan Model Testing Techniques. Journal of Aircraft, Vol. 34, No. 4, 1997, pp. 500-505.

${ }^{10}$ Milholen II, W. E., Chokani, N., and McGhee. R. J., Development of Semispan Model Test Techniques. Journal of Aircraft, Vol. 33, No. 6, 1996, pp. 1115-1122.

${ }^{11}$ Hemsch, M. J., "Development and Status of Data Quality Assurance Program at NASA Langley Research Center ... Toward National Standards," AlAA Paper 96-2214, June 1996.

${ }^{12}$ Hernsch, M., Grubb, J., Krieger, W., Cler, D., "Langley Wind Tunnel Data Quality Assurance - Check Standard Results," AIAA Paper 00-2201, June 2000.

${ }^{13}$ Burner, A., Liu, T., Garg, S., Ghee, T., Taylor, N., "Aeroelastic Deformation Measurement Technique for Slotted Flaps on Wind Tunnel Models," AlAA-Paper 00-2386, June 2000.

${ }^{14}$ Maskell, E. C., “A Theory of Blockage Effects on Bluff Bodies and Stalled Wings in a Closed Wind Tunnel," R\&M 3400, November 1963.

${ }^{15}$ Hackett, J. E., "Tunnel-Induced Gradients and Their Effect on Drag," Lockheed Georgia Co., Lockheed Engineering Rept. LG83ER0108 Revision 1, Marietta GA, September 1994.

${ }^{16}$ Iyer, V., Everhart, J. L., Bir, P. J., and Ulbrich, N., "Implementation of the WICS Wall-Interference Correction System at the National Transonic Facility," AIAA Paper 20002383 , June 2000

${ }^{17}$ Walker, E. L., Everhart, J. L., and lyer, V, "Sensitivity Study of the Wall Interference Correction System (WICS) for Rectangular Tunnels", AIAA Paper 01-0159, January 2001.

${ }^{18}$ Hemsch, M. J., and Luckring, J. M., "Connection Between Leading-Edge Sweep, Vortex Lift, and Vortex Strength for Delta Wings," AIAA J. Aircraft, Vol. 27, No. 5, May 1990. 
${ }^{19}$ Polhamus, E. C., "A Concept of the Vortex Lift of SharpEdged Delta Wings Based on a Leading-Edge Suction Analogy," NASA TN D-3767, 1966.

${ }^{20}$ Hemsch, M. J., Private communication

${ }^{21}$ Henderson, W. P., "Study of Various Factors Affecting Drag Due to Lift at Subsonic Speeds," NASA TN D-3584, October 1966. 